\title{
BMJ Open Safety and efficacy of mycophenolate mofetil in treating neuromyelitis optica spectrum disorders: a protocol for systematic review and meta-analysis
}

\author{
Mengyu Han (D) , ${ }^{1,2}$ Luqi Nong, ${ }^{1,2}$ Ziqiang Liu, ${ }^{1,2}$ You Chen, ${ }^{2}$ Yang Chen, ${ }^{1}$ \\ Huan Meng, ${ }^{1,2}$ Yali Qin, ${ }^{3}$ Zhijun Wang, ${ }^{2}$ Ming $\mathrm{Jin}^{2}$
}

To cite: Han M, Nong L, Liu Z, et al. Safety and efficacy of mycophenolate mofetil in treating neuromyelitis optica spectrum disorders: a protocol for systematic review and meta-analysis. BMJ Open 2020;10:e040371. doi:10.1136/ bmjopen-2020-040371

- Prepublication history for this paper is available online. To view these files, please visit the journal online (http://dx.doi. org/10.1136/bmjopen-2020040371).

Received 12 May 2020 Revised 12 0ctober 2020 Accepted 08 November 2020

Check for updates

(c) Author(s) (or their employer(s)) 2020. Re-use permitted under CC BY-NC. No commercial re-use. See rights and permissions. Published by BMJ.

${ }^{1}$ Graduate School, Beijing University of Chinese Medicine, Beijing, China

${ }^{2}$ Department of Ophthalmology, China-Japan Friendship Hospital, Beijing, China ${ }^{3}$ Zhongshan Ophthalmic Center, Sun Yat-Sen University, Guangzhou, China

Correspondence to

Dr Ming Jin;

jinmingyk@163.com

\section{ABSTRACT}

Introduction Neuromyelitis optica spectrum disorders (NMOSD) is an inflammatory and heterogeneous astrocyte disorder of the central nervous system with the characteristic of higher incidence in women and Asian people. Most patients with NMOSD have a course of recurrence and remission that is prone to cause paralysis and blindness. Several studies have confirmed the efficacy and promising prospect of mycophenolate mofetil (MMF) in the treatment of NMOSD. Yet its therapeutic effect and safety are controversial. Although there has been two published literature that is relevant to the topic of this study, both of them have certain defects, and they can only provide answers about the efficacy or safety of MMF in the treatment of NMOSD from partial perspectives or conclusions. This research aims to perform a direct and comprehensive systematic review and meta-analysis to evaluate MMF's effectiveness and safety in treating NMOSD

Methods and analysis This systematic review will cover all comparative researches, from randomised controlled trials to cohort studies, and case-control study. A relevant literature search will be conducted in PubMed, Web of Science, EMBASE, the Cochrane Library, China National Knowledge Infrastructure, Wanfang Database, China Science and Technology Journal Database and Chinese Biomedical Literature Database from their inception to 31 June 2020. We will also search registers of clinical trials, potential grey literature and abstracts from conferences. There are no limits on language and publication status. The reporting quality and risk of bias will be assessed by two researchers independently. Expanded Disability Status Scales and annualised relapse rate will be evaluated as the primary outcome. The secondary outcomes will consist of the frequency and severity of adverse events, best-corrected visual acuity, relapse-free rate and time to the next attack. A meta-analysis will be performed using RevMan V.5.3 software provided by the Cochrane Collaboration and Stata V.12.0.

Ethics and dissemination Because the data used for this systematic review will be exclusively extracted from published studies, ethical approval and informed consent of patients will not be required. The systematic review will be published in a peer-reviewed journal, presented at conferences and will be shared on social media platforms. PROSPERO registration number CRD42020164179.
Strengths and limitations of this study

- This study will carry out an exhaustive literature search to identify studies aimed at evaluating the efficacy and safety of mycophenolate mofetil in treating neuromyelitis optica spectrum disorders.

- One limitation of this study is that differences in patients, interventions and primary outcomes may mean that meta-analysis cannot be performed and there are plans for narrative and meta-analytical syntheses.

- Although we will include studies published in any language, translation difficulties may arise, which will result in the exclusion of these studies.

- The analysis of various sources of heterogeneity and the assessment of risk of bias of the included studies are a critical point for extracting and synthesising evidence-based conclusions.

\section{INTRODUCTION}

Neuromyelitis optica (NMO), also known as Devic disease, is generally considered to be a rare autoimmune astrocyte disorder of the central nervous system, induced by autoantibodies, dominated by humoral immunity and involving numerous immune cells and factors, with optic neuritis $(\mathrm{ON})$ and acute transverse myelitis as typical clinical manifestations. ${ }^{1}$ NMO has been known as a subtype of multiple sclerosis (MS) for over 100 years since it was first described and reported. ${ }^{2}$ Until 2004 , the discovery and confirmation of antiaquaporin-4 immunoglobulin G (AQP4-IgG) had made substantial progress in pathogenesis, diagnosis and treatment of NMO. ${ }^{34}$ The notion of neuromyelitis optica spectrum disorders (NMOSD) was first proposed based on the wide clinical use of specific AQP4-IgG, which mainly referred to the minimal AQP4-IgG positive NMO. However, the deficiencies of the diagnostic criteria of NMO in 2006 and NMOSD in 2007 became prominent 
with the incremental improvement of the specificity of clinical AQP4-IgG tests. In 2015, a groundbreaking international diagnostic protocol for NMOSD was put forward by the global NMO diagnostic team. ${ }^{5}$ NMOSD consists of NMO, ON, longitudinally extensive transverse myelitis and other common cerebral demyelinating syndromes. ${ }^{5}$ There are so far no reliable statistics on the worldwide incidence and prevalence of NMOSD. According to the current epidemiological evidence of small samples, the high incidence of this disease is among middle-aged and young women, with the onset age varying from 32 to 41 years old, and the incidence in women is about 10 times that of men. ${ }^{5}$ The incidence and prevalence of approximately $0.05-0.40$ and $0.52-4.40 / 100000$ vary from region to region. ${ }^{6} \mathrm{~A}$ populous region of Asia is the region with a high incidence of NMOSD ${ }^{7-9}$ Most patients with NMOSD have a recurrence and remission including $\mathrm{ON}$, myelitis, and lesions in special parts of the brain that are vulnerable to cause paralysis and blindness. ${ }^{5}$ NMOSD has become one of the most common causes of non-traumatic disability and blindness in young and middle-aged individuals, putting heavy burdens on the life, work and study, as well as the society and economy of various countries. ${ }^{10}$ Clinical studies indicate that approximately $1 / 4$ of patients will not be able to walk independently after an average of 5 years of NMO, approximately $10 \%$ will be wheelchair-dependent, and more than half of patients will have serious vision loss in at least one eye. ${ }^{11}$ In particular, ON associated with NMO (NMO-ON) possesses poor recovery even after traditional therapy, which often progresses into significant bilateral visual loss in the long term, leaving behind varying degrees of optic atrophy, which is different from MS. ${ }^{1213}$

Currently, there are no standardised guidelines for the clinical management of NMOSD. The class of NMOSD drugs is commonly referred to as disease-modifying drugs, ${ }^{14}$ and the treatment is split into two stages: the acute phase and the period of remission. The former is based on corticosteroids to reduce the severity and frequency of acute attacks that include intravenous corticosteroids, plasma exchange and immunoglobulin. Immunosuppressive agents, including mycophenolate mofetil (MMF), azathioprine (AZA), cyclophosphamide, methotrexate, mitoxantrone, tacrolimus, cyclosporine and monoclonal antibodies, are frequently used during the process of recovery to avoid recurrence and to mitigate the progression of neurological impairment. ${ }^{15} 16$ Although AZA and rituximab (RTX) are recommended as first-line therapies obtained from clinical trials and expert opinion from the published guidelines for NMOSD,${ }^{16}$ there are still adverse events (AEs) such as disease recurrence and myelosuppression that results in drug withdrawal or replacement of these drugs in patients with NMOSD. ${ }^{17}$ Other AEs for RTX have also been reported in recent years such as infusion reactions, infection and even death, ${ }^{18-20}$ and its clinical application has been constrained by such factors as high price. ${ }^{1821}$ Therefore, a better immunosuppressant for the treatment of
NMOSD is urgently needed. The application of MMF in NMOSD is still under investigation and is recommended as second-line treatments, ${ }^{16}$ but some studies have verified MMF's efficacy and promising potential, ${ }^{21-24}$ and only a few AEs were published. ${ }^{21}{ }^{22}$ Especially, additional studies have also indicated that MMF was more effective and triggered less AEs than AZA. ${ }^{25}{ }^{26}$ In patients experiencing AEs or poor response to AZA, MMF is recommended as an alternative therapy. ${ }^{16}$

Although MMF is increasingly employed in NMOSD, there is still controversy about its related harms and benefits. At present, there are mainly two published articles that are relevant to the topic and purpose of our research. ${ }^{27}{ }^{28}$ Nevertheless, these two studies have some imperfections in the direct evaluation of the efficacy and safety of MMF in the treatment of patients with NMOSD. For example, the Espiritu and Pasco paper did not quantitatively evaluate the efficacy of MMF in the treatment of NMOSD and did not compare the AEs of MMF with other drugs in the treatment of NMOSD. Additionally, Huang $e t a l$ 's research was a network meta-analysis and the literature related to MMF in this paper was three observational studies that made the number of included studies and closed loops per comparison were few, which might lower the reliability of the findings. In our study, the database we searched includes not only the English database but also the Chinese database. The retrieval time is limited to June 2020, and we will add three retrospective studies involving 471 patients with NMOSD, ${ }^{29-31}$ which makes the retrieval literature more comprehensive. At the same time, the conclusions of the previously published literature about the clinical effect of MMF were inconsistent. Poupart et al argued that RTX was clinically better tolerated than MMF. ${ }^{30}$ But Huang et $a l^{27}$ argued that MMF had the best drug tolerance and was superior to RTX. We expect our research to help solve this problem as well.

\section{METHODS}

This protocol has been registered on PROSPERO. Our protocol will follow the Meta-analysis of Observational Studies in Epidemiology, ${ }^{32}$ the Cochrane Handbook for Systematic Reviews of Interventions and the Preferred Reporting Items for Systematic Reviews and Meta-Analysis Protocol (PRISMA-P) statement guidelines. ${ }^{33} 34$

\section{Inclusion criteria for study selection}

Types of studies

All comparative researches, from randomised controlled trials (RCTs) to cohort studies, and case-control study, covering at least two interventions, will be included. The current clinical trial results will be objectively integrated, which is conducive to the evaluation of the efficacy and safety of MMF for NMOSD. We will exclude reviews, qualitative studies, animal trials, laboratory studies and studies only involving one intervention. 


\section{Types of patients}

Patients diagnosed as having NMOSD will be included in this study. ${ }^{535}$ There will be no restrictions based on other conditions, such as age at onset, sex, ethnicity, educational or economic status, number of pretreatment relapses, previous treatment, duration of illness, disease severity, baseline Expanded Disability Status Scales (EDSS) and AQP4-IgG serological status.

\section{Types of interventions}

Trials comparing MMF with placebo or any other active substances, including AZA, cyclophosphamide, methotrexate, mitoxantrone, tacrolimus, cyclosporine and monoclonal antibodies, will be considered. Besides, the types, dosage and frequency of MMF were not limited. Studies that MMF with combination therapy fail to objectively evaluate the efficacy and safety of MMF will be eliminated.

\section{Types of outcome measures}

\section{Primary outcomes}

1. EDSS: disability progression was characterised as an increase in the Kurtzke EDSS by at least 1 point above the pretreatment score if baseline score $<5.5$, and by at least half-point if baseline score $>5.5$. Outcome measured was the mean changes of EDSS before and after MMF treatment. ${ }^{3637}$

2. Annualised relapse rate (ARR): relapse is equivalent to a neurological symptom lasting for $>24$ hours, which occurs at least 30 days after the onset of a preceding event. ARR is computed as the number of relapses divided by the time in years (days). Post-treatment ARR was contrasted with pretreatment ARR. ${ }^{38}$

\section{Secondary outcomes}

1. The frequency and extent of AEs: during treatment and follow-up periods, any symptomatic events which had a possible, probable or definite causal relationship to MMF treatment were defined as AEs.

2. Relapse-free rate: the absence of relapse during the observation period of the study reported as percentage per study. ${ }^{35}$

3. Best-corrected visual acuity (BCVA): BCVA was measured using a standardised test, such as the Early Treatment Diabetic Retinopathy Study chart, Snellen chart or similar method, and other visual acuity measures would be allowed if findings could be justified as well as validated concerning accepted relevant standard measures. Outcome measured was the mean change of BCVA from before and after MMF treatment. ${ }^{39}$

4. Time to the next attack.

\section{Security index}

The safety was assessed by the occurrence of AEs. Any unexpected events that occurred during the studies will be recorded on an AEs report form, including ${ }^{28}$ :

1. General physical examination (temperature, pulse, respiration, blood pressure).

2. Routine examination of blood, urine and stool.

3. Liver and kidney function examination.
Table 1 Search strategy used in PubMed database

\begin{tabular}{ll}
\hline Number & Search terms \\
\hline$\# 1$ & (“Neuromyelitis Optica”[Mesh]) OR \\
& (((((neuromyelitis optica spectrum disorders [Title/ \\
& Abstract]) OR Devic Neuromyelitis Optica [Title/ \\
& Abstract]) OR Devic's Neuromyelitis Optica [Title/ \\
& Abstract]) OR Devic's Syndrome [Title/Abstract]) \\
& OR NMO spectrum disorders [Title/Abstract]) \\
& (“Mycophenolic Acid”[Mesh]) OR \\
& ((((Mycophenolate Mofetil [Title/Abstract]) OR \\
& "Mofetil,Mycophenolate” [Title/Abstract]) OR \\
& Cellcept [Title/Abstract]) OR Myfortic [Title/ \\
& Abstract]) OR RS61443 [Title/Abstract]) \\
& \#1 and \#2 \\
&
\end{tabular}

4. Gastrointestinal discomfort.

5. Hair loss or alopecia.

6. Allergic or anaphylactoid reactions.

7. Drug discontinued due to drug-related AEs.

8. Possible AEs and related detection indicators.

\section{Search methods for the identification of studies}

\section{Electronic searches}

A relevant literature search by sensitive search strategies was conducted using the following electronic databases from their inception to 31 June 2020: PubMed, Web of Science, EMBASE, the Cochrane Library, China National Knowledge Infrastructure, Wanfang Database, China Science and Technology Journal Database and Chinese Biomedical Literature Database. Search methods of Medical Subject Headings terms with free words were applied in English databases. The related terms are as follows: Participants (neuromyelitis optica, neuromyelitis optica spectrum disorders, Devic Neuromyelitis Optica, Devic's Neuromyelitis Optica, Devic's Syndrome, NMO spectrum disorders), Intervention (mycophenolic acid, mycophenolate mofetil, 'mofetil, mycophenolate', cellcept, myfortic, RS61443). The search strategy for PubMed is described in table 1, which will include all search terms, and other searches will be carried out based on those results. This will be suitably adapted to search in the other databases. There are no limits on language and publication status.

\section{Searching other resources}

We will also search PROSPERO, the International Clinical Trials Registry Platform, ClinicalTrials.gov, dissertations, and grey literature to identify systematic reviews or clinical trials related to MMF and NMOSD. Manual searches will be conducted for related journals and conference processes. We will also review papers and bibliographies included in the trials.

\section{Data collection and analysis \\ Selection of studies}

Two reviewers (MH and ZL) will independently browse the titles and abstracts of all of the retrieved records to distinguish and exclude any irrelevant articles. Studies 


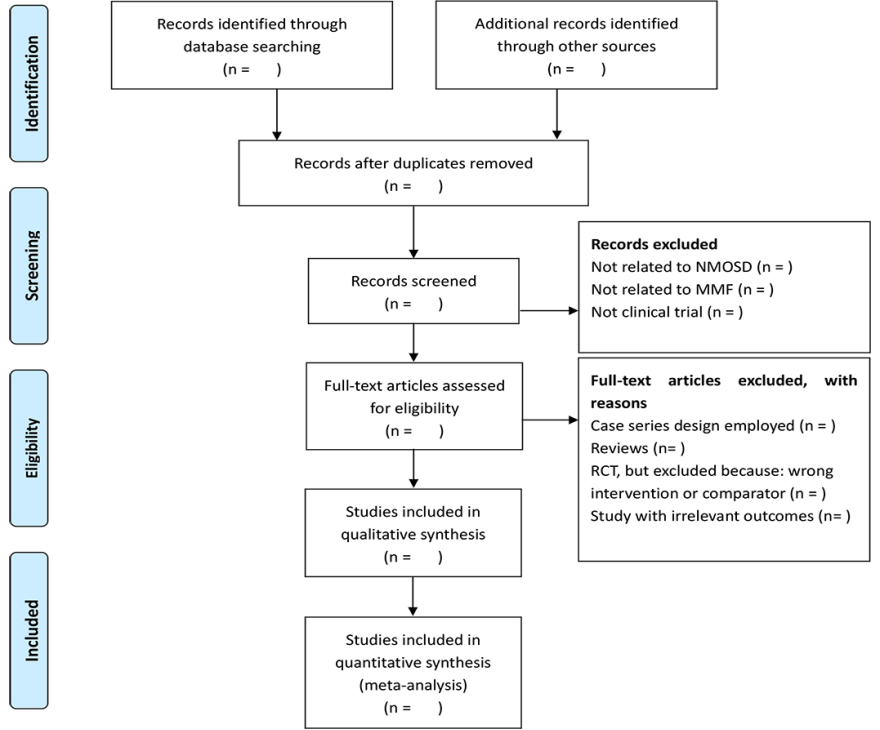

Figure 1 The PRISMA flow chart of the selection process. MMF, mycophenolate mofetil; NMOSD, neuromyelitis optica spectrum disorders; PRISMA, Preferred Reporting Items for Systematic Reviews and Meta-Analyses; RCT, randomised controlled trial.

only related to human subjects are to be included. Any discord will be resolved by discussion between the two authors and an arbiter (MJ). The selection procedure for the study is shown in a PRISMA flow chart (figure 1).

\section{Data extraction and management}

Based on the inclusion criteria, a standard form of data collection will be produced prior to data extraction. Search results will be entered into an EndNote V.X9 database and duplicate entries removed. Two authors (MH and ZL) will extract the data of interest from the eligible study and enter the data extraction sheet as follows: the basic characteristics of each study (study design or methods, author, title, source/journal, time of publication, country, hospital setting); participants' characteristics (average age, gender, sample size, inclusion and exclusion criteria, baseline situation); interventions (type, duration, frequency and dosage of MMF, randomisation, allocation concealment, blinding methods); comparators (AZA, tacrolimus, cyclosporine, monoclonal antibodies, placebo and so on); outcomes (measures, main outcomes, security indexes and follow-up); if funded, it will also be recorded. When the consensus on data extraction is not available through discussion, the third reviewer (MJ) will make a decision.

\section{Assessment of risk of bias}

Two authors (YangC and LN) will independently estimate the risk and bias using the Cochrane risk of bias (ROB) assessment tool for RCTs. ${ }^{40}$ Methodological quality evaluation of the included observational studies will be carried out using the Newcastle-Ottawa Scale. ${ }^{41}$ The RevMan software program (V.5.3) will document the selected details of each study. ${ }^{42}$
Measures of treatment effect

The risk ratio and $95 \%$ CI will be used to analyse dichotomous data and calculate the treatment effect. A weighted mean difference or a standard mean difference with $95 \%$ CIs will be used to analyse continuous outcomes.

\section{Unit of analysis issue}

We will only extract the first experimental period data of crossover trials to avoid carryover effects. In the meantime, given that there are multiple intervention groups in trials, we will combine all analogous groups into a single pairwise comparison to avoid a unit of analysis issue.

\section{Management of missing data}

Reviewers (YQ and YouC) will contact the appropriate author of the included trials for clarification or more details via email and telephone if necessary. The missing data will be deleted, if there is no response from the author. That will be addressed in the discussion in this case. If quantitative data were not available, then the qualitative analysis should be used.

\section{Assessment of heterogeneity and data synthesis}

We will use all of the case data for the analysis data. Heterogeneity will be tested with a standard $\mathrm{X}^{2}$ test. ${ }^{43}$ To quantify the impact of the statistical heterogeneity on the systematic review, the $\mathrm{I}^{2}$ value will be applied to calculate and present the heterogeneity degree. If $\mathrm{p}>0.1, \mathrm{I}^{2}<50 \%$, it is considered that there is no heterogeneity between the trials, and the model of fixed effect will be used, otherwise, the model of random effect will be adopted. All statistical analyses will be performed using the RevMan V.5.3 software provided by the Cochrane Collaboration, using the software to obtain forest plots and test the heterogeneity between the included studies. The Grades of Recommendation, Assessment, Development and Evaluation will be used to assess the meta-analysis findings and determine the quality of evidence. Where meta-analysis may not be feasible due to lack of clinical trials or heterogeneity, systematic narrative synthesis will be adopted.

\section{Assessment of reporting biases}

When 10 or more studies are included in a meta-analysis, we will evaluate funnel plot asymmetry for reporting biases and small-study effects using Egger's method. ${ }^{44}$ For Egger's test, $p$ value of greater than 0.05 was determined as no significant publishing bias or small-study effects in studies. As funnel plot asymmetry does not necessarily suggest reporting bias, we will attempt to recognise potential causes for the asymmetry, including poor methodological quality and true heterogeneity of studies.

\section{Subgroup analysis}

On detection of heterogeneity, a subgroup analysis will be carried out to judge the source of heterogeneity. The criteria for a subgroup analysis are as follows:

1. Age.

2. Type of MMF.

3. Research type. 
4. Participation population.

5 . Type of control interventions.

6. Intervention dosage, frequency and duration.

7. AQP4-IgG serological status.

Sensitivity analysis

The ROB tool will be used to estimate methodological quality in the case of sufficient data from trials. Sensitivity analysis will be performed to determine the robustness of aggregate estimates and to detect whether any single study accounts for a substantial proportion of heterogeneity by eliminating the included studies from the summary review one by one. If low-quality articles are deleted, then a second meta-analysis will be carried out. Comparison and discussion of the results and effect size of the two meta-analyses will be held. ${ }^{45}$

\section{Patient and public involvement}

Patients and/or the public will not participate in the study. However, once scientific publications disseminate our findings, they are circulated across social networks so that our conclusions will affect the actions of neuroophthalmologists and health policymakers.

\section{Ethics and dissemination}

Because the data used for this systematic review will be exclusively extracted from published studies, ethical approval and informed consent of patients will not be required. The systematic review will be published in a peer-reviewed journal, presented at conferences and will be shared on social media platforms.

\section{DISCUSSION}

Pathogenesis, diagnosis and treatment of NMO are rapidly growing areas of research as AQP4-IgG were first identified. Patients with NMOSD should receive standardised and personalised immunotherapy as soon as possible, as any further acute episodes may result in severe and often irreversible disability. The challenges in discovering new and better drugs for NMO are the rareness of the disease and the unfavourable prognosis in many cases, which make clinical studies with placebo groups difficult. ${ }^{16}$ Many studies have confirmed the efficacy and promising prospect of MMF in the treatment of NMOSD,${ }^{21-24}$ and only a few AEs were reported. ${ }^{21}{ }^{22}$ Additional studies have also indicated that MMF was more effective and triggered less AEs than AZA. ${ }^{25}{ }^{26}$ However, its therapeutic effect and safety remain controversial. Although there has been two published literature that is relevant to the topic of this study, ${ }^{27}$ both of them have certain defects, and they can only provide answers about the efficacy or safety of MMF in the treatment of NMOSD from partial perspectives or conclusions. If our paper is completed, it will be a currently searchable protocol for a traditional meta and systematic review that directly and synthetically evaluates the efficacy and safety of MMF in the treatment of NMOSD. One of the strengths of this protocol will use a comprehensive search strategy of published literature. The overall data used in each analysis will be evaluated qualitatively and quantitatively. The sources of heterogeneity and different subgroups of the articles will be analysed to comprehensively evaluate the efficacy and safety of MMF in the treatment of NMOSD, and to increase the credibility of the article content and conclusions. We expect that this systematic review will benefit patients with NMOSD, physicians, healthcare administrators and policymakers.

Contributors $\mathrm{MH}$ conceived and designed the protocol, and drafted the protocol manuscript. MH developed the search strategy, with input from ZL and LN. MH and ZL planned data extraction. MH, YangC and ZW planned the quality appraisal of all included studies. MH, ZL, LN, YangC, HM, YouC, ZW, YQ and MJ critically revised the manuscript for methodological and intellectual content. All authors approved the final version. Additional contributions are as follows: MH, ZL, ZW and MJ—conceptualisation. MH, ZL, LN and HM-data curation. MH and ZL-formal analysis. MH_-funding acquisition. MJ—investigation. MH, ZL, LN and YouCmethodology. MJ—project administration. MH, ZL, LN and MJ—resources. MH, ZL and $\mathrm{LN}$ - software. MH, ZW and MJ—supervision. HM, YangC, YQ and MJvalidation. HM, YangC, YQ and MJ—visualisation. MH, ZL, LN and MJ—writing (original draft). MH, ZL, LN and MJ—writing (review and editing).

Funding This study was funded by the National Natural Science Foundation of China (No. 81574029).

Disclaimer The funders had no role in the design, execution or writing of the study. Competing interests None declared.

Patient and public involvement Patients and/or the public were not involved in the design, or conduct, or reporting, or dissemination plans of this research.

Patient consent for publication Not required.

Provenance and peer review Not commissioned; externally peer reviewed.

Open access This is an open access article distributed in accordance with the Creative Commons Attribution Non Commercial (CC BY-NC 4.0) license, which permits others to distribute, remix, adapt, build upon this work non-commercially, and license their derivative works on different terms, provided the original work is properly cited, appropriate credit is given, any changes made indicated, and the use is non-commercial. See: http://creativecommons.org/licenses/by-nc/4.0/.

ORCID iD

Mengyu Han http://orcid.org/0000-0003-2342-268X

\section{REFERENCES}

1 Jasiak-Zatonska M, Kalinowska-Lyszczarz A, Michalak S, et al. The immunology of neuromyelitis Optica-Current knowledge, clinical implications, controversies and future perspectives. Int J Mol Sci 2016;17:273.

2 Jarius S, Wildemann B. On the contribution of Thomas Clifford Allbutt, F.R.S., to the early history of neuromyelitis optica. $J$ Neurol 2013;260:100-4.

3 Yang X, Ransom BR, Ma J-F. The role of AQP4 in neuromyelitis optica: more answers, more questions. J Neuroimmunol 2016;298:63-70.

4 Wingerchuk DM, Lennon VA, Lucchinetti CF, et al. The spectrum of neuromyelitis optica. Lancet Neurol 2007;6:805-15.

5 Wingerchuk DM, Banwell B, Bennett JL, et al. International consensus diagnostic criteria for neuromyelitis optica spectrum disorders. Neurology 2015;85:177-89.

6 Pandit L, Asgari N, Apiwattanakul M, et al. Demographic and clinical features of neuromyelitis optica: a review. Mult Scler 2015;21:845-53.

7 Bukhari W, Prain KM, Waters P, et al. Incidence and prevalence of NMOSD in Australia and New Zealand. J Neurol Neurosurg Psychiatry 2017;88:632-8.

8 Viswanathan S, Wah LM, WallL M. A nationwide epidemiological study on the prevalence of multiple sclerosis and neuromyelitis optica spectrum disorder with important multi-ethnic differences in Malaysia. Mult Scler 2019;25:1452-61. 
9 Miyamoto K, Fujihara K, Kira J-I, et al. Nationwide epidemiological study of neuromyelitis optica in Japan. J Neurol Neurosurg Psychiatry 2018;89:667-8.

10 Wingerchuk DM, Hogancamp WF, O'Brien PC, et al. The clinical course of neuromyelitis optica (Devic's syndrome). Neurology 1999;53:1107.

11 Jiao Y, Fryer JP, Lennon VA, et al. Updated estimate of AQP4-IgG serostatus and disability outcome in neuromyelitis optica. Neurology 2013;81:1197-204.

12 Drori T, Chapman J. Diagnosis and classification of neuromyelitis optica (Devic's syndrome). Autoimmun Rev 2014;13:531-3.

13 Optic Neuritis Study Group. Visual function 15 years after optic neuritis: a final follow-up report from the optic neuritis treatment trial. Ophthalmology 2008;115:1079-82.

14 Papadopoulos MC, Bennett JL, Verkman AS. Treatment of neuromyelitis optica: state-of-the-art and emerging therapies. Nat Rev Neurol 2014;10:493-506.

15 Wu Y, Zhong L, Geng J. Neuromyelitis optica spectrum disorder: pathogenesis, treatment, and experimental models. Mult Scler Relat Disord 2019;27:412-8.

16 Trebst $\mathrm{C}$, Jarius S, Berthele A, et al. Update on the diagnosis and treatment of neuromyelitis optica: recommendations of the neuromyelitis optica Study Group (NEMOS). J Neurol 2014;261:1-16.

17 Elsone L, Kitley J, Luppe S, et al. Long-term efficacy, tolerability and retention rate of azathioprine in 103 aquaporin-4 antibodypositive neuromyelitis optica spectrum disorder patients: a multicentre retrospective observational study from the UK. Mult Scler 2014;20:1533-40.

18 Torres J, Pruitt A, Balcer L, et al. Analysis of the treatment of neuromyelitis optica. J Neurol Sci 2015;351:31-5.

19 Annovazzi P, Capobianco M, Moiola L, et al. Rituximab in the treatment of neuromyelitis optica: a multicentre Italian observational study. J Neurol 2016;263:1727-35.

20 Collongues N, de Seze J. An update on the evidence for the efficacy and safety of rituximab in the management of neuromyelitis optica. Ther Adv Neurol Disord 2016;9:180-8.

21 Jacob A, Matiello M, Weinshenker BG, et al. Treatment of neuromyelitis optica with mycophenolate mofetil: retrospective analysis of 24 patients. Arch Neurol 2009;66:1128-33.

22 Huh S-Y, Kim S-H, Hyun J-W, et al. Mycophenolate mofetil in the treatment of neuromyelitis optica spectrum disorder. JAMA Neurol 2014;71:1372-8

23 Chen $\mathrm{H}$, Zhang $\mathrm{Y}$, Shi Z, et al. The efficacy and tolerability of mycophenolate mofetil in treating neuromyelitis optica and neuromyelitis optica spectrum disorder in Western China. Clin Neuropharmacol 2016;39:81-7.

24 Jiao Y, Cui L, Zhang W, et al. Dose effects of mycophenolate mofetil in Chinese patients with neuromyelitis optica spectrum disorders: a case series study. BMC Neurol 2018;18:47.

25 Chen H, Qiu W, Zhang Q, et al. Comparisons of the efficacy and tolerability of mycophenolate mofetil and azathioprine as treatments for neuromyelitis optica and neuromyelitis optica spectrum disorder. Eur J Neurol 2017;24:219-26.

26 Yang Y, Wang C-J, Wang B-J, et al. Comparison of efficacy and tolerability of azathioprine, mycophenolate mofetil, and lower dosages of rituximab among patients with neuromyelitis optica spectrum disorder. J Neurol Sci 2018;385:192-7.
27 Huang W, Wang L, Zhang B, et al. Effectiveness and tolerability of immunosuppressants and monoclonal antibodies in preventive treatment of neuromyelitis optica spectrum disorders: a systematic review and network meta-analysis. Mult Scler Relat Disord 2019;35:246-52.

28 Espiritu Al, Pasco PMD. Efficacy and tolerability of azathioprine for neuromyelitis optica spectrum disorder: a systematic review and meta-analysis. Mult Scler Relat Disord 2019;33:22-32.

29 Zhou Y, Zhong X, Shu Y, et al. Clinical course, treatment responses and outcomes in Chinese paediatric neuromyelitis optica spectrum disorder. Mult Scler Relat Disord 2019;28:213-20.

30 Poupart J, Giovannelli J, Deschamps R, et al. Evaluation of efficacy and tolerability of first-line therapies in NMOSD. Neurology 2020;94:e1645-56.

31 Shi Z, Du Q, Chen $\mathrm{H}$, et al. Effects of immunotherapies and prognostic predictors in neuromyelitis optica spectrum disorder: a prospective cohort study. J Neurol 2020;267:913-24.

32 Stroup DF, Berlin JA, Morton SC, et al. Meta-analysis of observational studies in epidemiology: a proposal for reporting. JAMA;283:2008-12.

33 Shamseer L, Moher D, Clarke M, et al. Preferred reporting items for systematic review and meta-analysis protocols (PRISMA-P) 2015 elaboration and explanation. BMJ 2015;349:7647.

34 Moher D, Shamseer L, Clarke M, et al. Preferred reporting items for systematic review and meta-analysis protocols (PRISMA-P) 2015 statement. Syst Rev 2015;4:1.

35 Wingerchuk DM, Lennon VA, Pittock SJ, et al. Revised diagnostic criteria for neuromyelitis optica. Neurology 2006;66:1485-9.

36 Kurtzke JF. Rating neurologic impairment in multiple sclerosis: an expanded disability status scale (EDSS). Neurology 1983;33:1444-52.

37 Enriquez CAG, Espiritu AI, Pasco PMD. Efficacy and tolerability of mitoxantrone for neuromyelitis optica spectrum disorder: a systematic review. J Neuroimmunol 2019;332:126-34.

38 Lavery AM, Verhey LH, Waldman AT. Outcome measures in relapsing-remitting multiple sclerosis: capturing disability and disease progression in clinical trials. Mult Scler Int 2014;2014:262350

39 Squires H, Poku E, Bermejo I, et al. A systematic review and economic evaluation of adalimumab and dexamethasone for treating non-infectious intermediate uveitis, posterior uveitis or panuveitis in adults. Health Technol Assess 2017;21:1-170.

40 Savović J, Weeks L, Sterne JA, et al. Evaluation of the Cochrane collaboration's tool for assessing the risk of bias in randomized trials: focus groups, online survey, proposed recommendations and their implementation. Syst Rev 2014;15:37.

41 Wells G, Shea B, O'Connell D, et al. The Newcastle-Ottawa scale (NOS) for assessing the quality of nonrandomised studies in metaanalyses. Mult Scler;13:968-74.

42 The Nordic Cochrane collaboration. Review manager (RevMan). 5.0. Copenhagen: The Nordic Cochrane collaboration, 2008.

43 Chen B, Benedetti A. Quantifying heterogeneity in individua participant data meta-analysis with binary outcomes. Syst Rev 2017;6:243

44 Egger M, Davey Smith G, Schneider M, et al. Bias in meta-analysis detected by a simple, graphical test. BMJ 1997;315:629-34.

45 Shih KC, Lun CN, Jhanji V, et al. Systematic review of randomized controlled trials in the treatment of dry eye disease in Sjogren syndrome. J Inflamm 2017;14:26. 